\title{
Interactive comment on "Deciphering the evolution of the Bleis Marscha rock glacier (Val d'Err, eastern Switzerland) with cosmogenic nuclide exposure dating, aerial image correlation, and finite-element modelling" by Dominik Amschwand et al.
}

Jakob Heyman (Referee)

jakob.heyman@gu.se

Received and published: 10 September 2020

\section{General comments}

The paper presents a study of a rock glacier in eastern Switzerland combining cosmogenic dating, recent velocity estimation from aerial imagery, and simulation of the rock glacier deformation in an effort to investigate and reconstruct the post-glacial rock glacier evolution. The questions addressed appear relevant for The Cryosphere and 
the study presents a novel and interesting concept combining multiple chronological and numerical techniques. The paper is clearly presented and it is mostly easy to understand. I find the paper highly interesting and mostly very good. There are however a number of issues, primarily related to the interpretation of the exposure ages, that should be addressed before publication. In particular, several interpretations of the exposure ages appear to be not supported by the data, and for some interpretations the underlying assumptions have not been spelled out. In the specific comments and technical corrections below these issues (and other more minor issues) are presented in more detail.

\section{Specific comments}

1. Exposure age interpretations 1 In section 4.2, you write: "The exposure ages in general anticorrelate with elevation and correlate with down-flow distance". I agree with this but an important part here is "in general". A few lines further down you write "The boulders remained exposed at the surface and were passively transported at the rock glacier surface between talus and top of front slope [-]. We conclude that the exposure ages on the active lobe have negligible systematic errors and no overall age shift from inheritance/pre-exposure (systematically "too old") or nuclide loss/incomplete exposure (systematically "too young")". This statement is in my mind incorrect and it should be changed. It is clear from Figure 6 that a maximum of three out of seven samples overlap within uncertainties with the linear regression line. This implies that at least four out of the seven samples (plus sample Err8 and Err11 which are not included in the linear regression) have experienced either prior or incomplete exposure. The absolute numbers of the age "error" are small $(<1 \mathrm{ka})$ but the relative errors are not. For example, sample Err6 has an exposure age that is less than half of the age expected from the linear regression. Such an error cannot be seen as "negligible" if trying to use the age to constrain the velocity of the rock glacier. I suggest that you are more open with the fact that the samples may have experienced both prior and incomplete exposure and that it is really difficult to identify how much each of these

Printer-friendly version

Discussion paper
Interactive comment 
processes have affected the exposure ages. If assuming that the linear regression actually represent the rock glacier transport an underlying assumption is that prior and incomplete exposure have affected the group of exposure ages by an equal amount. This assumption should be properly spelled out in the text. It is easy to argue that both prior and incomplete exposure may very well have affected the exposure ages. We will likely get prior exposure unless the rock-wall erosion is really deep/rapid and we can easily get incomplete exposure if the rocks on top of the rock glacier move around and topple every now and then. The scatter of exposure ages around the regression line could perhaps be used to try to estimate an uncertainty of the rock glacier velocity.

2. Exposure age interpretations 2 Further down in section 4.2, you write: "the 10Be ages of Err2 and Err15 are significantly younger than the further up-slope sampled Err14. It is unlikely that this apparent age inversion reflects internal creep, as the laminar flow behaviour of a rock glacier makes mixing impossible and contradicts our finding of linear relationship between exposure age and down-flow distance on the active lobes". I agree with the statement about samples Err2 and Err15. However, just the same can be said about samples Err6 and Err7 on the active lobe which are younger than samples Err5 and Err9 further up-slope. The difference in the interpretation of the exposure ages on the active lobe and the inactive lobe is strange and not supported by the data, and I think that it should be changed.

3. Exposure age interpretation 3 In section 5.1 (L 517-518) your write about Figure 10: "The exposure ages for the samples located on the presently active upper lobes are slightly less than the travel times by about $10-100$ years, which is within the uncertainty margins". This statement is wrong and it should be changed. Of the seven samples falling below the 1:1 line, only one sample (Err5) overlap with the line. You further write: "The measured surface velocity field is representative, and the discrepancy is smaller the closer the samples are to the central flow line (Err3, 4, 5, 10), where the influence of lateral viscous drag is smallest." It is correct that the age discrepancy is smaller for samples 3,4,5,10 compared to samples 6,7,9, but the age discrepancy is still large for

Printer-friendly version

Discussion paper
Interactive comment 
samples Err3 and Err4. Sample Err3 has an exposure age of 229 years compared to an estimated travel time of 800 years. Sample Err4 has an exposure age of 556 years compared to an estimated travel time of 1000 years. The statement about the discrepancy should be changed.

4. Exposure age interpretation 4 Further down in section 5.1 (L 529-531), you write: "Our data on the active lobe of Bleis Marscha rock glacier show that the exposure ages for an undisturbed rock-glacier lobe can be addressed as (minimum) travel times. While travelling during the active phase, boulders remain mutually interlocked within the clastsupported framework and the age dispersion remains small. Notably, it also shows that pre-travel nuclide build-up, inheritance, and pre-exposure in the rock wall or on the talus are insignificant." These statements are poorly supported by the data and they should be changed. The age dispersion is NOT small for the question of rock glacier velocity (several hundred years for multiple samples!). As the ages DON'T cluster and overlap with the estimated travel times, there MUST have been some prior and/or incomplete exposure causing the exposure age scatter (unless the scatter comes from the measurements or some other source of error).

5. Exposure age interpretation 5 In lines 550-553 you write: "Intermittent boulder instability during inactivation has been affecting the nuclide inventory of each boulder individually, leading to incomplete exposure by self-shielding and nuclide loss by weathering or spalling, and ultimately to tendentially underestimated true exposure times ('apparent rejuvenation'). This results in a large dispersion of exposure ages and exposure age inversions on the low-elevation rock-glacier lobes." I largely agree with these statements about the exposure ages from the inactive part of the rock glacier. However, we cannot really be sure that NO prior exposure has affected the exposure ages. Further, from Figure 10 it is clear that if lifting up the 1:1 line the samples from the inactive part could fit the line much better than the samples on the active part. You never discuss this fact in the manuscript and I think that you should. You could fit a regression line to the inactive samples in the same way as to the active samples and 
my guess is that you would get a similarly good match to the data. I imagine that such an approach could potentially be used to try to estimate the velocity of the presently inactive part during its active phase (although this will of course involve several questionable assumptions...). In any case, I think you should at least mention more clearly the correlation of exposure age against down-flow distance also on the inactive part of the rock glacier and try to offer some explanation for it.

Interactive

comment

6. Exposure age interpretation 6 In lines 564-568 you write: "We find that exposure ages need to be interpreted according to the dynamic history of the sampled rockglacier lobe. On undisturbed lobes where the current surface creep is concordant with the micro-topography, exposure ages represent (minimum) travel times. This applies to active lobes without destabilisation. On 'discordant' lobes where microtopography reflects different stress conditions and must have undergone disruptive dynamic changes, the exposure ages represent time elapsed since the major dynamic change. Inactivation or destabilisation are such disruptive events." I find these statements too categorical and not really well-supported by the data. First, if the statement about undisturbed lobes is correct and the estimated $36 \mathrm{Cl}$ age of sample Err 8 is roughly correct, the minimum travel time of the active lobe is significantly longer than what you state elsewhere in the manuscript. Second, if the statement about 'discordant' lobes is correct, the "major dynamic change" must have been in the period 8.9 to $4.8 \mathrm{ka}$. That is a long period (c. 4000 years) for a "disruptive event". Third, with data from one specific rock glacier it seems a bit too bold to draw conclusions about exposure dating of rock glaciers in general (which is how I interpret text).

7. L 135 "using the CRONUS-EARTH online calculator". You should state which version of the calculator you have used. Further, I would suggest using the updated version 3 calculator and perhaps also use the Chironico 10Be reference production rate directly instead of the northeast North America production rate. In the v. 3 calculator you can pick the Chironico input data from the ICE-D database to calibrate the production rate and then use that to calculate exposure ages. Perhaps you should also

Printer-friendly version

Discussion paper 
exclude the sample with the lowest $10 \mathrm{Be}$ concentration because with that included you get a P-value of only 0.033 . However, all this is not really important for the outcome so just disregard it if you don't want to do the extra work.

8. Table 1. I suggest changing "calibrated to the 07KNSTD standard" to "calibrated to the S2007N standard" because that is what is stated in section 3.2. I also suggest that you add the down-profile distance for each sample that you use in Figure 6. That data is not included anywhere else in the manuscript and I think that it should be included somewhere.

9. L 673-674 "The oldest exposure age (Err11) atop the front of the active upper lobe indicates that the most recent phase of activity phase likely began at or just before $2.8 \pm 0.1 \mathrm{ka}$ and lasts until today." This statement is correct only under the assumption that the sample has not experienced prior or incomplete exposure, and that assumption should be clearly expressed. See also lines 751-752 where you write almost exactly the same but "before 2.8" instead of "at or just before 2.8".

10. L 756-757 "Furthermore, travel time estimates from streamline interpolations agree well with the exposure ages." This is incorrect - see points 3-4 above.

11. L 771-772 "Code availability. The code used for flowline and strain-rate calculations is available by request from the corresponding author." Why not upload the code to a repository and add a link to it or include it as a supplement? That would make it easier for anyone who is interested in the code.

Technical corrections

12. L 13 I suggest changing "The results suggest" to "The results indicate".

13. L 65-66 "Long-term effects on rock glacier development must remain unresolved (Kenner and Magnusson, 2017)" This sentence sounds a bit strange to me (in particular "must remain") and I suggest rephrasing it.

14. L 68-69 "Cosmogenic radionuclides exposure dating is a unique tool because it 
directly measures a chronometric, numerical exposure age of the landform surface". I suggest rephrasing this. Exposure dating does not directly measure an exposure age - it measures the cosmogenic nuclide concentration which we can use to calculate an exposure age (involving several critical assumptions).

15. L 74 "at an elevation range of 2400-2700 m a.s.I. Previous studies". I don't know what is correct writing here, but it should perhaps be one more punctuation mark after "m a.s.l." to show that the sentence ends.

16. L 116 I suggest changing "the surface exposure dating method" to "surface exposure dating".

17. Figure 2 Disregard these comments if you disagree (they regard the visual impression and are not really important). In figure a, the text with the white edges does not look good so I suggest trying to use just white text or to make the white edges thinner or perhaps bright grey. In figure b, I suggest making and $\mu$ smaller (they look too big). It also looks a bit strange with having "surface", "boundaries", and "base" underlined.

18. Figure 3 I suggest adding units I to $V$ to this map. For the three texts with white background, I suggest trying to make the white edges thinner or perhaps bright grey.

19. Figure 4 It is a bit strange to have this map shifted 90 degrees compared to Figure 3 and I would probably try to keep the same orientation in both figures (although this is not really important so disregard this comment if you wish). For the black texts with white background, I suggest trying to make the white edges thinner or perhaps bright grey.

20. Figure 5 The text in the photos is in several cases a bit difficult to read. I suggest trying to change the black text with white background to just white text or perhaps white text with black or grey background (but thinner background text than in the present version).

Printer-friendly version

21. $L 331$ "ages ranging from $\sim 4.8$ to $\sim 9.5 \mathrm{ka}$. The age $9.5 \mathrm{ka}$ here seems to be too 
high as the oldest age is $8.9 \mathrm{ka}$.

22. L 504-505 "The active high-elevation lobes (2550-2700 $\mathrm{m}$ a.s.l.) with surface speeds up to $60 \mathrm{~cm}$ a-1 display clustered exposure ages within $0.23 \pm 0.04$ to $2.8 \pm$ $0.1 \mathrm{ka}$ ". The exposure ages on the active lobe should NOT be called "clustered". See major points 1 and 2 above.

23. Figure 10. You state in the figure caption that the exposure age uncertainties are the "analytical uncertainties (internal errors)". Although it won't really matter for the outcome, you should rather use the external uncertainties including production rate uncertainty as you are comparing the exposure ages with travel time estimates that are independent from the exposure ages.

Interactive comment on The Cryosphere Discuss., https://doi.org/10.5194/tc-2020-209, 2020. 